\title{
Urban-rural differences in catastrophic health expenditure among households with chronic non- communicable disease patients : evidence from China Family Panel Studies
}

\author{
Xianzhi Fu ( $1371810134 @ q q . c o m)$ \\ Wuhan University \\ Qi-wei Sun \\ Provincial Higher People's court in Heilongjiang \\ Chang-qing Sun \\ Zhengzhou University \\ Fei Xu \\ Zhengzhou University \\ Jun-jian He \\ Zhengzhou University
}

\section{Research}

Keywords: Catastrophic health expenditure, Fairlie nonlinear decomposition, Blinder-Oaxaca decomposition, China

Posted Date: June 25th, 2020

DOI: https://doi.org/10.21203/rs.3.rs-37742/v1

License: (c) (i) This work is licensed under a Creative Commons Attribution 4.0 International License.

Read Full License

Version of Record: A version of this preprint was published at BMC Public Health on May 6th, 2021. See the published version at https://doi.org/10.1186/s12889-021-10887-6. 
1 Urban-rural differences in catastrophic health expenditure

2 among households with chronic non-communicable disease

3 patients: evidence from China Family Panel Studies

4 Title:

5 Urban-rural differences in catastrophic health expenditure among households

6 with chronic non-communicable disease patients: evidence from China Family

7 Panel Studies

8 Authors:

9 Xian-zhi Fu ${ }^{1 *}$ Ph.D. student; Qi-wei Sun ${ }^{2}$ LLM; Chang-qing Sun ${ }^{3}$ Ph.D.; Fei Xu ${ }^{3}$

10 Ph.D. student; Jun-jian $\mathrm{He}^{3} \mathrm{MPH}$

\section{Authors E-mail:}

12 Xian-zhi Fu*: 1371810134@qq.com

13 Qi-wei Sun: 2427057938@qq.com

14 Chang-qing Sun: zzugwsy@163.com

15 Fei Xu: phare@gs.zzu.edu.cn

16 Jun-jian He: hh510968@163.com

\section{Authors affiliations:}

$18{ }^{1}$ School of Political Science and Public Administration, Wuhan University,

19 Wuhan 430072, Hubei, China.

$20{ }^{2}$ Provincial Higher People's Court, Harbin 150000, Heilongjiang, China. 
$1{ }^{3}$ Department of Social Medicine and Health Management, College of Public

2 Health, Zhengzhou University, Zhengzhou 450001, Henan, China.

$3 *$ Corresponding author

$4 \quad$ Ph.D. student Xian-zhi Fu

5 School of Political Science and Public Administration, Wuhan University,

$6 \quad$ Wuhan 430072, Hubei, China;

7 Phone: +86 13595954776;

$8 \quad$ E-mail: $1371810134 @ q q . c o m$

9

10

11

12

13

14

15

16

17

18

19

20

21

22 


\section{Abstract}

2 Background: The prevalence of chronic non-communicable diseases

3 (NCDs) challenges the Chinese health system reform. Little is known

4 for the differences in catastrophic health expenditure (CHE) between

5 urban and rural households with NCD patients. This study aims to

6 measure the differences above and quantify the contribution of each

7 variable in explaining the urban-rural differences.

8 Methods: The second and the fourth waves of the China Family Panel

9 Studies (CFPS) data, conducted in 2012 and 2016, were employed in 10 this cross-sectional study. The techniques of Fairlie nonlinear

11 decomposition and Blinder-Oaxaca decomposition were employed to

12 measure the contribution of each independent variable to the urban-

13 rural differences.

14 Results: The CHE incidence and intensity of households with NCD 15 patients were significantly higher in rural areas than in urban areas. 16 The explained disparity of CHE incidence increased from 3.15\% in 172012 to $27.04 \%$ in 2016, and the corresponding values of $\mathrm{CHE}$ 18 intensity rose from $21.30 \%$ in 2012 to $53.37 \%$ in 2016 . The major 19 contribution to the urban-rural differences in CHE was associated 20 with household economic status, education level, health status and 21 supplementary medical insurance (SMI). 
1 Conclusions: Compared with urban households with NCD patients,

2 rural households with NCD patients have higher risk of incurring CHE

3 and heavier economic burden of diseases. Policy interventions should

4 give priority to decreasing the urban-rural disparity in observable 5 characteristics.

6 Keywords: Catastrophic health expenditure, Fairlie nonlinear

7 decomposition, Blinder-Oaxaca decomposition, China.

\section{1. Background}

Achieving universal health coverage, defined as ensuring that all 10 people have access to essential health services without suffering 11 financial constraints by 2030 , is one of the key targets of the 12 sustainable development goals (SDGs) [1, 2]. However, a global 13 monitoring report released by the WHO and World Bank reflects the 14 situation of "poverty caused by illness" in the global population in 15 2017: (1) more than 122 million people were classified as "poor" 16 (living on less than $\$ 3.10$ a day) due to health care expenditure; (2) 17 about 100 million people were pushed into "extremely poor" (living 18 on less than $\$ 1.90$ a day) because they have to pay for health care [3].

19 With the prevalence of chronic non-communicable diseases (NCDs) 20 accompanied by accelerated population aging, increasing number of 21 individuals worldwide will suffer from catastrophic health 
1 expenditure (CHE) in the future.

As the global epicenter of NCDs epidemic, China is under great

3 pressure. A 2005 study estimated that NCDs had become the leading

4 cause of death and disease burden in China, accounting for $80 \%$ of

5 deaths and $70 \%$ of disability-adjusted life-years lost [4]. In 2015,

6 NCDs contributed to $86.6 \%$ of all deaths and $70 \%$ of the total disease

7 burden in China [5]. The heavy burden of NCDs has greatly increased

8 the economic risks for many vulnerable groups in China.

9 The fundamental functions of a health system is not only to 10 promote access to essential health care services, but also to improve 11 the ability of households to withstand the financial catastrophe 12 associated with illness [6]. The Chinese health system has been 13 working to protect vulnerable households against CHE. In 2009, 14 China's new round of health system reform involved a series of policy 15 measures, including the reduction of out-of-pocket (OOP) medical expenditure and expansion of basic health care coverage by 2020 [7,

17 8]. Three types of basic medical insurance schemes, including the 18 Urban Employee Basic Medical Insurance, Urban Residents Basic 19 Medical Insurance and New Rural Cooperative Medical Scheme, have 20 been established to decrease the financial burden of NCDs on 21 households. In 2013, more than 95\% of residents were covered by 
1 basic medical insurance in China, which was a sign of universal

2 coverage of basic medical insurance $[9,10]$. In addition,

3 supplementary medical insurance (SMI), including commercial

4 medical insurance, public servant medical subsidy, enterprise

5 supplementary medical subsidy, employee medical subsidy for large

6 medical expenses, and employee mutual medical insurance, was

7 established to meet the needs of residents for multiple levels of

8 health services [11]. However, there was still evidence that medical

9 expenditure due to NCDs played an important role in the main causes

10 of poverty among rural households in China [12]. As NCDs are

11 characterized by long treatment duration and high treatment costs

12 [13], substantial financial hardships create obstacles to health

13 services utilization for rural households with NCD patients in China,

14 leading to further escalation of health problems. Therefore, it is

15 necessary and urgent to pay attention to the CHE among rural

16 households with NCD patients.

17 Several researches have investigated the financial catastrophe 18 among individuals or households suffering from NCDs around the 19 world. Three existing studies emphasized that households with NCD 20 patients were in the high risk to incur CHE in China, Korea and Iran $21[9,14,15]$. Gwatidzo (2017) found that adults aged 50 or above in 
1 India were less likely to incur CHE due to diabetes mellitus

2 medication use compared to China [16]. Zhao (2019) identified that

3 the CHE incidence among rural households with NCD patients

4 notably exceeded the average level of urban households with NCD

5 patients in China [17]. Xie (2017) verified the main reasons why

6 households with members suffering from NCDs in rural China were

7 prone to CHE [18]. To sum up, most of the studies have explored the

8 CHE of households with NCD patients in rural areas of a country or in

9 a whole country. However, there is still a lack of discussion on the

10 urban-rural differences in CHE among households with NCD patients

11 and its influencing factors. In addition, understanding the urban-rural

12 differences in the financial risks of NCD medical expenses and the

13 factors related to the differences can prompt more effective efforts to

14 reduce the economic risk of rural households with NCD patients.

15 The objectives of this study were as follows: (1) to measure the 16 extent of CHE for urban and rural households with NCD patients, (2)

17 to examine the urban-rural differences in the degree of CHE between

18 the two groups, and (3) to quantify the contribution of each variable 19 to the urban-rural differences.

\section{Methods}


This study was based on a publicly available database, the

3 China Family Panel Studies (CFPS), which was conducted by the

$4 \quad$ Institute of Social Science Survey (ISSS) of Peking University every

5 two years from 2010 to 2016. The CFPS used a three-stage,

6 stratified, probability-proportional-to-scale (PPS) random sampling

7 method to select sample from twenty-five provinces in China. It

8 was representative that the sample of CFPS representing $94.5 \%$ of

9 the population in mainland China [19]. The questionnaire for CFPS

10 involved a wide range of variables, such as demography

11 characteristics, socioeconomic status, health status, health services

12 utilization, family relationships and medical insurance and so on.

13 We used the second and the fourth waves of cross-sectional

14 data from CFPS, which involved 13,315 households in 2012 and 1514,019 households in 2016, respectively. The inclusion criteria for

16 the interviewed households were as follows: (1) having completed

17 information; and (2) having members with NCDs. In this survey,

18 NCDs were determined by whether a respondent had been

19 diagnosed by a doctor within the previous six months? Finally, 202,871 households with NCD patients in 2012 and 4,065 households 21 with NCD patients in 2016 were specialized in this study, including 
$1 \quad 1,348$ households in urban areas and 1,523 households in rural

2 areas in 2012, and 1,982 households in urban areas and 2,083

3 households in rural areas in 2016.

4 2.2. Measurement of CHE

5 We referred to the studies of Wagstaff and van Doorslaer to

6 determine the relevant indicators of measuring CHE [20, 21]. OOP

7 medical expenditure only included direct medical expenditure made

8 by any household members, and excluded indirect expenditure

9 related to seeking health services (e.g., transportation, food, 10 accommodation, lost productivity due to illness). Since the

11 substitution of non-food household expenditure for total household 12 expenditure partly avoided the measurement deviations that were 13 often overlooked in poor households, we used non-food household 14 expenditure as the denominator to calculate CHE $[22,23]$. The non-

15 food expenditure of a household is defined as the portion of total 16 household expenditure excluding food household expenditure. 17 According to exiting literature [17, 22, 24, 25], the threshold for CHE 18 was defined as $40 \%$. More specifically, if OOP medical expenditure of 19 a household exceeded $40 \%$ of its non-food household expenditure, 20 the household was classified as incurring CHE. A binary variable was 21 defined to determine whether a household experienced CHE or not, 
1 as shown in formula (1):

$$
E_{i}=\left\{\begin{array}{l}
0 \text { if } \frac{T_{i}}{\left(x_{i}-f_{i}\right)}<\text { threshold } \\
1 \text { if } \frac{T_{i}}{\left(x_{i}-f_{i}\right)} \geq \text { threshold }
\end{array}\right.
$$

2 where $T_{i}$ means the OOP medical expenditure of household $i, x_{i}$ 3 is the total expenditure of household $i, f_{i}$ stands for the food 4 expenditure of household $i$, and threshold is defined as $40 \%$. The 5 calculation of CHE incidence and intensity can be specified as below:

$$
\begin{gathered}
H=\frac{1}{N} \sum_{i=1}^{N} E_{i} \\
O=\frac{1}{N} \sum_{i=1}^{N} E_{i}\left(\frac{T_{i}}{\left(x_{i}-f_{i}\right)}-z\right)=\frac{1}{N} \sum_{i=1}^{N} O_{i} \\
M P O=\frac{O}{H}
\end{gathered}
$$

6 where $N$ represents the total sample size, $H$ means the CHE 7 incidence in the overall sample. CHE intensity is estimated by 8 overshoot and mean positive overshoot (MPO). $O$ stands for 9 overshoot, which is the average percentage of OOP medical 10 expenditure that exceeds a given threshold in the overall sample [26].

11 MPO indicates the average percentage of OOP medical expenditure in 12 excess of the threshold among households incurring CHE [20]. The 13 higher values of overshoot and MPO both stand for heavier financial 
1 burden of diseases for the household.

2 2.3. Definitions of independent variables

3 Referring to the previous reports, we included the

4 characteristics of each household and its household head into the

5 regression model as independent variables [22, 23, 27-29].

6 Households characteristics involved eight variables: the annual

7 household income per capita, household size, receiving inpatient

8 services, having members below 5 years old, having elderly members,

9 having members covered by basic medical insurance, having 10 members covered by SMI, and geographic location. The

11 characteristics of household head involved four variables: gender, 12 education, marriage, and self-assessed health status. We used the 13 natural logarithm of the annual household income per capita to 14 measure economic status of a household. Table 1 presents the 15 detailed descriptions of the above independent variables.

\subsection{Methodology}

17 The Blinder-Oaxaca decomposition technique, proposed by 18 Blinder and Oaxaca [30, 31], was applied in this study to analyze the 19 contribution of each independent variable to the urban-rural 20 differences in CHE. The implementation of decomposition analysis 
1 needs to be based on the relationship between CHE and a series of

2 independent variables.

As CHE incidence $\left(E_{i}\right)$ is a binary variable, probit model is

4 applied to estimate the effect of the independent variables on the

5 CHE incidence. The specific regression model is shown below:

$$
Y^{\gamma}=F\left(X^{\gamma} \beta^{\gamma}\right)
$$

6 where $F$ represents the cumulative distribution function of the

7 standard normal distribution, superscript $\gamma$ represents the rural or

8 urban households, $Y$ is the CHE incidence, $X$ stands for the

9 independent variables, and $\beta$ denotes the regression coefficient.

10 Fairlie extended the technique of Blinder-Oaxaca decomposition

11 to the application of nonlinear model [32, 33]. Given the probit

12 regression model is a nonlinear regression model, this study

13 employed the method of Fairlie nonlinear decomposition to

14 decompose the urban-rural differences in CHE incidence between

15 two groups into two components:

$$
\begin{aligned}
\bar{Y}^{R}-\bar{Y}^{U}= & \underbrace{\left[\sum_{i=1}^{N^{R}} \frac{F\left(X_{i}^{R} \beta^{R}\right)}{N^{R}}-\sum_{i=1}^{N^{U}} \frac{F\left(X_{i}^{U} \beta^{R}\right)}{N^{U}}\right]}_{\text {Explained part }} \\
& +\underbrace{\left[\sum_{i=1}^{N^{U}} \frac{F\left(X_{i}^{U} \beta^{R}\right)}{N^{U}}-\sum_{i=1}^{N^{U}} \frac{F\left(X_{i}^{U} \beta^{U}\right)}{N^{U}}\right]}_{\text {Unexplained part }}
\end{aligned}
$$


1 Where superscript $\mathrm{R}$ represents the rural households,

2 superscript $\mathrm{U}$ means the urban households. $\bar{Y}$ does not necessarily

3 equal $F(\bar{X} \beta)$. The first term in formula (6) stands for the explained

4 part of the urban-rural differences between two groups, which is

5 caused by the disparity in distribution of independent variables, and

6 the second term represents the unexplained part due to the disparity

7 in regression coefficient [34].

8 The detailed decomposition involves a natural one-to-one

9 matching of cases between the two groups to identify the

10 contribution of independent variables. The subsample was drawn

11 from the majority group (rural households), and matched the

12 minority group (urban households) based on the ranking of CHE

13 incidence. The contribution of variable $X_{1}$ to the urban-rural

14 differences in CHE incidence is estimated as follows:

$$
\frac{1}{N^{U}} \sum_{i=1}^{N^{U}} F\left(\alpha^{*}+X_{1 i}^{R} \beta_{1}^{*}+X_{2 i}^{R} \beta_{2}^{*}\right)-F\left(\alpha^{*}+X_{1 i}^{U} \beta_{1}^{*}+X_{2 i}^{R} \beta_{2}^{*}\right)
$$

Where $\beta^{*}$ stands for the regression coefficient from the probit model for the overall sample. It should be noted that the results are

17 sensitive to the order of independent variables in the decomposition 18 of nonlinear model [34]. Following Fairlie [33], independent 19 variables were randomly ordered in the decomposition of nonlinear 
1 model. This study repeated the above steps 1000 times to obtain the

2 average value of decomposition results, representing the

3 contribution of each independent variable.

4 Similarly, the contribution of $X_{2}$ to the urban-rural differences in

5 CHE incidence is calculated as follows:

$$
\frac{1}{N^{U}} \sum_{i=1}^{N^{U}} F\left(\alpha^{*}+X_{1 i}^{U} \beta_{1}^{*}+X_{2 i}^{R} \beta_{2}^{*}\right)-F\left(\alpha^{*}+X_{1 i}^{U} \beta_{1}^{*}+X_{2 i}^{U} \beta_{2}^{*}\right)
$$

6 In addition, since the CHE intensity $\left(O_{i}\right)$ is a continuous variable,

7 multiple linear regression is used to analyze the factors affecting the

8 CHE intensity. The specific regression model can be written as:

$$
Y^{\gamma}=X^{\gamma} \beta^{\gamma}+\varepsilon^{\gamma}
$$

9 where $Y$ represents the CHE intensity, $X$ stands for a vector of 10 independent variables, $\beta$ is a vector of regression coefficient

11 including intercept, and $\varepsilon$ denotes the random error term. The contribution of each independent variable to the urban-

13 rural differences in CHE intensity between two groups was divided 14 into two components using two-fold Blinder-Oaxaca decomposition 15 approach [35, 36]:

$$
\bar{Y}^{R}-\bar{Y}^{U}=\underbrace{\left(\bar{X}^{R}-\bar{X}^{U}\right) \beta^{*}}_{\text {Explained part }}+\underbrace{\left[\bar{X}^{R}\left(\beta^{R}-\beta^{*}\right)+\bar{X}^{U}\left(\beta^{*}-\beta^{U}\right)\right]}_{\text {Unexplained part }}
$$


1 linear regression for the overall sample, $\bar{X}$ represents the

2 corresponding covariate means of the independent variables. The

3 first term indicates the explained part, representing the contribution

4 attributable to group disparity in distribution of independent

5 variables, and the second term indicates the unexplained part,

6 representing the contribution attributable to group disparity in

7 regression coefficient.

8 All statistical analyses were performed in STATA software

9 version 15.1 , and $\mathrm{p}<0.05$ was considered statistically significant.

10 3. Results

11 3.1. Descriptive statistics

12 Table 2 shows the summary statistics for general 13 characteristics of the urban and rural households with NCD 14 patients. The annual household income per capita of urban and 15 rural households were 18,513.86 CNY and 9,538.07 CNY in 2012, 16 rising to 29,905.45 CNY and 16,110.23 CNY in 2016. In both 2012 17 and 2016, the mean household size in rural areas was greater than 18 that in urban areas. Meanwhile, the rural households had higher 19 probability in receiving inpatient services in the last 12 months, 20 having children below 5 years old, having elderly members, 
1 having basic medical insurance, and having married household

2 head than urban households. With respect to the coverage of SMI,

3 the proportions of households having SMI were higher in urban

4 areas in comparison with the rural areas in 2012 and 2016. The

5 percentages of households having female household head were

6 higher in urban areas than in rural areas. The proportion of urban

7 households located in the east is the highest, and the percentages

8 of rural households located in the west is the highest. Among

9 urban households, household heads of education achievement of

10 high school and above had the highest proportion, while among

11 rural households, household heads of education level of illiterate

12 had the highest percentage.

14 3.2. CHE incidence and intensity.

15 Table 3 illustrates CHE incidence and intensity of urban and 16 rural households with NCD patients. In 2016, $19.88 \%$ of 17 households in urban areas experienced CHE. Meanwhile, the 18 overshoot of urban households was $4.39 \%$ in 2016, suggesting 19 that the average percentage of OOP medical expenditure that 20 exceeded the given threshold over all urban households was $214.39 \%$. The MPO for urban households was $22.08 \%$ in 2016, 
meaning that if the burden of overshoot was divided equally by all urban households incurring CHE, the average extent of exceeding given threshold was $22.08 \%$. Each of the other row could be interpreted in a similar pattern for rural/urban households with

\subsection{Associated factors of CHE incidence.}

Table 4 presents the probit regression results for factors associated with the CHE incidence in urban and rural households with NCD patients. Household size and education attainment of household head significantly decreased the CHE incidence, while receiving inpatient services in the last 12 months and having elderly members significantly increased the occurrence of exposure to CHE. Economic status was negatively correlated with CHE incidence, but the correlation was not statistically significant in the sample of rural households in 2012. The geographic location of central and west significantly reduced the CHE incidence of rural households in 2016. Meanwhile, the geographic location of west was negatively correlated with CHE incidence of rural households in 2012. Poor self-assessed health status of household head significantly increased the CHE incidence for urban households in 2012 and for rural households in 2016. Basic 
1 medical insurance and SMI did not affect CHE incidence at a

2 significant level.

3 3.4. Associated factors of CHE intensity.

4

5

6

7

8

9

The associated factors of the CHE intensity $\left(O_{i}\right)$ are shown in Table 5. Economic status and household size significantly reduced the CHE intensity, while receiving inpatient services in the last 12 months and having elderly members significantly increased the CHE intensity. The geographic location of central and west significantly decreased the CHE intensity of rural households in 2016. Meanwhile, the geographic location of west was negatively associated with CHE intensity of rural households in 2012. Education of household head was negatively associated with CHE intensity, but did not significantly affect the CHE intensity of rural households in 2012. Poor self-assessed health status of household head significantly increased the CHE intensity of rural households. Basic medical insurance and SMI did not affect CHE intensity at a significant level.

3.5. Aggregate decomposition. 
Table 6 displays the results for aggregate decomposition of

2 the urban-rural differences in CHE incidence and intensity $\left(O_{i}\right)$

3 among households with NCD patients.

4

The explained disparity of CHE incidence increased from 3.15\%

in 2012 to $27.04 \%$ in 2016, and the corresponding values of $\mathrm{CHE}$ intensity rose from $21.30 \%$ in 2012 to $53.37 \%$ in 2016.

7 3.6. Decomposition of contribution of all explanatory variables.

The urban-rural differences in CHE incidence and intensity $\left(O_{i}\right)$ among households with NCD patients is further decomposed into the contribution of each variable, as shown in Table 7 and $11 \quad$ Table 8.

With respect to the urban-rural differences in CHE incidence in 2012, the explained part was mainly attributed to household size (-31.49\%), geographic location (west, $-17.00 \%)$, and education of household head (middle school, 5.79\%; high school and above, 27.46\%). The main contribution to the explained disparity in CHE incidence in 2016 was associated with economic status $(29.80 \%)$, household size $(-33.22 \%)$, geographic location (west, -18.73\%), gender of household head (4.72\%), education of 
1 household head (middle school, 5.54\%), and self-assessed health

2 status of household head (poor, 19.06\%).

3

4

5

6

7

8

9

With regard to the explained disparity of CHE intensity in 2012, the main contributors were economic status (36.90\%), household size (-37.08\%), geographic location (west, -18.75\%), gender of household head (6.76\%), education of household head (primary school, -5.97\%; middle school, 4.46\%; high school and above, $21.62 \%$ ), and self-assessed health status of household head (poor, 6.62\%). In 2016, the explained disparity in CHE intensity was mainly associated with economic status (47.19\%), household size (-34.78\%), SMI (2.27\%), geographic location (west, $-13.82 \%)$, education of household head (primary school, -7.25\%; middle school, 4.72\%; high school and above, 28.54\%), and self-assessed health status of household head (poor, 11.91\%).

\section{Discussion:}

By analyzing the national representative cross-sectional data of two waves from the CFPS in 2012 and 2016, this study estimates the extent of CHE for urban and rural households with 
NCD patients, as well as the differences in the degree of CHE between the two groups.

Here, we found that the CHE incidence of households with NCD patients in urban and rural areas were $19.88 \%$ and $26.02 \%$, respectively, which are much higher than the results of another study on the overall proportion of households incurring CHE in China (urban households: 13.06\%; rural households: $17.70 \%$ ) [17]. It indicates that the risk tolerance of households with NCD patients to OOP medical expenditure is lower than the average level of Chinese households. Our results also showed that the households with NCD patients had higher incidence and intensity of CHE in rural areas than in urban areas, demonstrating that rural households with NCD patients have higher risk of incurring CHE and heavier economic burden of diseases.

Using regression analysis to examine the relevant influencing factors for CHE incidence and intensity in 2012 and 2016, this research identified several key determinants reported in prior studies (e.g., economic status, household size, having elderly members, education of household head, receiving inpatient services in the last 12 months) [10, 22, 23, 37]. Specifically, higher annual household income per capita, larger household size and 
higher education level of household head protected against CHE in urban and rural households with NCD patients. The geographic location of the west area reduced the risk of incurring CHE and financial burden of diseases in rural households with NCD patients. A potential explanation is that rural households with NCD patients in the western China forgo their needed health services due to the

low income [38]. Conversely, households with elderly members and poor self-assessed health status of household head had higher risk of incurring CHE and heavier economic burden of diseases.

Basic medical insurance did not significantly reduce the incidence and intensity of CHE in both two groups, which is consistent with some existing literature [11, 22, 39-41]. The weak effect of basic medical insurance in reducing the incidence and intensity of CHE could be attributed to the relatively lower level of scope and actual reimbursement rate, as well as the heavy economic burden of NCDs [23]. It implies that under the premise of universal medical insurance in China, the reimbursement rate and benefit package of basic medical insurance for NCDs should be further improved to alleviate the economic burden of NCDs.

As the supplementary form of basic medical insurance, the coverage of SMI did not efficiently decrease the risk of incurring 
1 CHE and alleviate financial burden of diseases. Given that SMI is

2 characterized by high reimbursement rate and voluntary 3 participation [42, 43], one plausible reason for the weak 4 performance of SMI is low coverage rate. The coverage rate of SMI 5 in urban households with NCD patients increased from $2.74 \%$ in 62012 to $5.80 \%$ in 2016, while the coverage rate of SMI in rural 7 households with NCD patients increased from 1.44\% in 2012 to $8 \quad 2.93 \%$ in 2016, indicating that a large number of households in $9 \quad$ China are not covered by SMI (Table 2).

From 2012 to 2016, the reduction of the unexplained disparity offset the increase of the explained disparity, resulting in a slight decrease of the rural-urban differences in the incidence and intensity of CHE.

More importantly, this article identified major contributors to explain the urban-rural differences in CHE incidence and intensity among households with NCD patients. Specifically, economic status made the largest positive contribution to the urban-rural differences. In addition, from 2012 to 2016, the disparity explained by economic status gradually increased, which can be attributed to the increase in the income gap between urban and rural households with NCD patients. Similarly, the education 

explained the disparity. Therefore, any intervention aimed at decreasing this disparity may be effective if they focus on the observable characteristics mentioned above.

Our research also found that SMI did not significantly affect the rural-urban differences in CHE incidence, but it was still a variable which was worthy of attention. The contribution of SMI to the rural-urban differences in CHE rose in 2016 compared with 
The study is not without its limitations. First, various

2 characteristics (e.g., the levels of medical institution, actual 3 reimbursement rate of medical insurance, distance to the nearest

5. Conclusion:

In conclusion, the present study suggested that rural households with NCD patients had higher CHE incidence and intensity than urban ones, and the rural-urban differences gradually decreased from 2012 to 2016. By using the methods of Fairlie nonlinear decomposition and Blinder-Oaxaca 
1 decomposition, this research found that the annual household

2 income per capita, education of household head, self-assessed

3 health status of household head and SMI explained the rural-

4 urban differences in CHE. Therefore, relevant policy interventions

5 should give priority to decreasing the urban-rural disparity in

6 observable characteristics mentioned above.

\section{Acknowledgments}

8 The authors sincerely thank Ju Sun and Qiang Yao for their

9 helpful suggestions and valuable comments.

\section{Funding}

11 This study is supported by the National Social Science

12 Foundation of China (Grant number: 15BSH043). The funders had no

13 role in the design of this study, or data collection, analysis, and 14 preparing the manuscript.

\section{Availability of data and materials}

The data source of this study was a publicly available database,

17 the China Family Panel Studies (CFPS), which was hosted by the 18 Institute of Social Science Survey (ISSS) of Peking University every 19 two years from 2010 to 2016. 


\section{Author Contributions}

2 Xian-zhi Fu formulated the primary framework of the study. Qi-

3 wei Sun and Fei Xu conducted data analysis. Jun-jian He and Chang-

4 qing Sun interpreted the results. All authors reviewed the manuscript.

5 Authors' information

6 Xian-zhi Fu, School of Political Science and Public Administration,

7 Wuhan University, Wuhan 430072, Hubei, China.

8 Qi-wei Sun, Provincial Higher People's Court, Harbin 150000, 9 Heilongjiang, China.

11 Management, College of Public Health, Zhengzhou University,

12 Zhengzhou 450001, Henan, China.

Fei $\mathrm{Xu}$, Department of Social Medicine and Health Management,

14 College of Public Health, Zhengzhou University, Zhengzhou 450001, 15 Henan, China.

16 Jun-jian He, Department of Social Medicine and Health

17 Management, College of Public Health, Zhengzhou University, 18 Zhengzhou 450001, Henan, China. 
2 of the institutional and national research committees and with the

31964 Helsinki Declaration and its subsequent revisions or similar

4 ethical standards. Each volunteer participant obtained a written

5 informed consent based on inclusion criteria.

\section{Consent for publication}

7 The authors give consent for publication of this paper in

8 International Journal for Equity in Health.

\section{Competing interests}

The authors declare that there are no competing interests.

\section{References}

1. Evans DB, Etienne C. Health systems financing and the path to universal coverage. Bull World Health Organ. 2010; 88:402.

2. Hogan DR, Stevens GA, Hosseinpoor AR, Boerma T. Monitoring universal health coverage within the Sustainable Development Goals: development and baseline data for an index of essential health services. Lancet Glob Health. 2018; 6:e152-e168.

3. World Health Organization-World Bank. Global Monitoring Report 2017: $\begin{array}{llll}\text { Tracking universal health } 2019 . & \text { coverage; }\end{array}$ http://www.emro.who.int/fr/media/ advocacy-materials/who-world-banktracking-universal-health-coverage-2017-global-monitoring-report.html

4. Wang L, Kong L, Wu F, Bai Y, Burton R. Preventing Chronic Diseases in China. Lancet. 2005; 366:1821-1824.

5. The National Health and Family Planning Commission of China. The progress of disease prevention in China (in Chinese). Capital Journal of Public Health. 2015; 9(3):97-101.

6. Reinhardt U E , Cheng T M. The world health report 2000 -- Health systems: improving performance. Bulletin of the World Health Organization. 2000; 78(8):1064. 
7. Guo Y, Shibuya K, Cheng G, Rao K, Lee L, Tang S. Tracking China's health reform. Lancet. 2010; 375:1056-1058.

8. Yip WCM, Hsiao WC, Chen W, Hu SL, Ma J, Maynard A. Early appraisal of China's huge and complex health-care reforms. Lancet. 2012; 379:833-842.

9. $\mathrm{Xu} \mathrm{Y,} \mathrm{Ma} \mathrm{J,} \mathrm{Wu} \mathrm{N,} \mathrm{Fan} \mathrm{X,} \mathrm{Zhang} \mathrm{T,} \mathrm{Zhou} \mathrm{Z,} \mathrm{Gao} \mathrm{J,} \mathrm{Ren} \mathrm{J,} \mathrm{Chen} \mathrm{G.}$ Catastrophic health expenditure in households with chronic disease patients: A pre-post comparison of the New Health Care Reform in Shaanxi Province, China. PLoS One. 2018; 13:e0194539.

10. Si YF, Zhou ZL, Su M, Wang X, Lan X, Wang D, Gong SQ, Xiao X, Shen C, Ren $Y L$, et al. Decomposing inequality in catastrophic health expenditure for selfreported hypertension household in Urban Shaanxi, China from 2008 to 2013: two waves' cross-sectional study. Bmj Open. 2019; 9:9.

11. Sun J, Lyu SJ. The effect of medical insurance on catastrophic health expenditure: evidence from China. Cost Effectiveness and Resource Allocation. 2020; 18:11.

12. Hui-ping Wang, Zeng-tao Wang, Peng-cheng Ma. Situation analysis and thinking about poverty caused by illness in rural areas: Based on research data of 1214 families whose poverty is caused by illness in 9 Provinces in West China (in Chinese). Economist. 2016; (10):71-81.

13. Fu XZ, Wang LK, Sun CQ, Wang DD, He JJ, Tang QX, Zhou QY. Inequity in inpatient services utilization: a longitudinal comparative analysis of middleaged and elderly patients with the chronic non-communicable diseases in China. International Journal for Equity in Health. 2020; 19:17.

14. Zhang XY, Xu QQ, Guo XL, Jing ZY, Sun L, Li JJ, Zhou CC. Catastrophic health expenditure: a comparative study between hypertensive patients with and without complication in rural Shandong, China. Bmc Public Health. 2020; $20: 13$.

15. Lee M, Yoon K. Catastrophic Health Expenditures and Its Inequality in Households with Cancer Patients: A Panel Study. Processes. 2019; 7:12.

16. Gwatidzo SD, Williams JS. Diabetes mellitus medication use and catastrophic healthcare expenditure among adults aged 50+years in China and India: results from the WHO study on global AGEing and adult health (SAGE). Bmc Geriatrics. 2017; 17:15.

17. Zhao Y, Oldenburg B, Mahal A, Lin Y, Tang S, Liu X. Trends and socioeconomic disparities in catastrophic health expenditure and health impoverishment in China: 2010 to 2016. Tropical Medicine \& International Health. 2020; 25:236-247.

18. Xie B, Huo MH, Wang ZQ, Chen YJ, Fu R, Liu MN, Meng Q. Impact of the New Cooperative Medical Scheme on the trend of catastrophic health expenditure in Chinese rural households: results from nationally representative surveys from 2003 to 2013. Bmj Open. 2018; 8:9.

19. Ma XC, Wang ZY, Liu XY. Progress on Catastrophic Health Expenditure in China: Evidence from China Family Panel Studies (CFPS) 2010 to 2016. International Journal of Environmental Research and Public Health. 2019; $16: 11$.

20. O'Donnell O, Doorslaer E, Wagstaff A, Lindelow M. Analyzing Health Equity Using Household Survey Data: A Guide to Techniques and Their Implementation. 2008.

21. Wagstaff $A$, Lindelow $M$. Can insurance increase financial risk? The curious case of health insurance in China. J Health Econ. 2008; 27:990-1005. 
22. Wang $\mathrm{ZH}$, Li XJ, Chen MS. Catastrophic health expenditures and its inequality in elderly households with chronic disease patients in China. International Journal for Equity in Health. 2015; 14:11.

23. Si Y, Zhou Z, Su M, Ma M, Xu Y, Heitner J. Catastrophic healthcare expenditure and its inequality for households with hypertension: evidence from the rural areas of Shaanxi Province in China. International journal for equity in health. 2017; 16:27-27.

24. Xu K, Evans DB, Kawabata K, Zeramdini R, Klavus J, Murray CJ. Household catastrophic health expenditure: a multicountry analysis. Lancet. 2003; 362:111-117.

25. Van Minh H, Kim Phuong NT, Saksena P, James CD, Xu K. Financial burden of household out-of pocket health expenditure in Viet Nam: findings from the National Living Standard Survey 2002-2010. Soc Sci Med. 2013; 96:258-263.

26. Ahmadnezhad E, Murphy A, Alvandi R, Abdi Z. The impact of health reform in Iran on catastrophic health expenditures: Equity and policy implications. Int J Health Plann Manage. 2019.

27. Kavosi Z, Rashidian A, Pourreza A, Majdzadeh R, Pourmalek F, Hosseinpour A, Mohammad $\mathrm{K}$. Inequality in household catastrophic healthcare expenditure in a low-income society of Iran. Health policy and planning. 2012; 27:613-623.

28. Arsenijevic J, Pavlova M, Rechel B, Groot W. Catastrophic Health Care Expenditure among Older People with Chronic Diseases in 15 European Countries. PloS one. 2016; 11:e0157765.

29. Lee JE, Shin HI, Do YK, Yang EJ. Catastrophic Health Expenditures for Households with Disabled Members: Evidence from the Korean Health Panel. Journal of Korean Medical Science. 2016; 31:336-344.

30. Blinder AS. WAGE DISCRIMINATION - REDUCED FORM AND STRUCTURAL ESTIMATES. Journal of Human Resources. 1973; 8:436-455.

31. Oaxaca R. Male-Female Wage Differentials in Urban Labor Markets. International Economic Review. 1973; 14:693-709.

32. Fairlie RW. The absence of the African-American owned business: An analysis of the dynamics of self-employment. Journal of Labor Economics. 1999; 17:80-108.

33. Fairlie R. An Extension of the Blinder-Oaxaca Decomposition Technique to Logit and Probit Models. Journal of Economic and Social Measurement. 2005; 30:305-316.

34. Mehta $H$, Rajan S, Aparasu $R$, Johnson M. Application of the nonlinear Blinder-Oaxaca decomposition to study racial/ethnic disparities in antiobesity medication use in the United States. Research in social \& administrative pharmacy. 2012; 9.

35. Jann B. A STATA implementation of the Blinder-Oaxaca decomposition. The Stata Journal. 2008; 8.

36. Oaxaca R, Ransom M. On Discrimination and the Decomposition of Wage Differentials. Journal of Econometrics. 1994; 61:5-21.

37. Xu Y, Gao J, Zhou Z, Xue Q, Yang J, Luo H, Li Y, Lai S, Chen G. Measurement and explanation of socioeconomic inequality in catastrophic health care expenditure: Evidence from the rural areas of Shaanxi Province. BMC health services research. 2015; 15:256.

38. Li Y, Wu Q, Liu C, Kang Z, Xie X, Yin H, Jiao M, Liu G, Hao Y, Ning N. Catastrophic Health Expenditure and Rural Household Impoverishment in China: What Role Does the New Cooperative Health Insurance Scheme Play? PloS one. 2014; 9:e93253. 
39. Jiang $\mathrm{CH}$, Ma JD, Zhang X, Luo WJ. Measuring financial protection for health in families with chronic conditions in Rural China. Bmc Public Health. 2012; $12: 7$.

40. Liu H, Zhao Z. Impact of China's Urban Resident Basic Medical Insurance on 5 Health Care Utilization and Expenditure. 2012.

41. Lei X, Lin W. The New Cooperative Medical Scheme in rural China: does more coverage mean more service and better health? Health economics. 2009; 18 Suppl 2:S25-46.

42. Dong K. Medical Insurance System Evolution in China. China Economic Review. 2009; 20:591-597.

43. Li AQ, Shi $Y L$, Yang $X$, Wang ZH. Effect of Critical Illness Insurance on Household Catastrophic Health Expenditure: The Latest Evidence from the National Health Service Survey in China. International Journal of Environmental Research and Public Health. 2019; 16:17.

44. Guo N, Iversen T, Lu MS, Wang J, Shi LW. Does the new cooperative medical scheme reduce inequality in catastrophic health expenditure in rural China? Bmc Health Services Research. 2016; 16:11. 